\title{
Infrared microscopic analysis of tissues: a comparison of methodologies.
}

\author{
M Jackson ${ }^{1}$, Janie Dubois ${ }^{1}$, Richard Baydak ${ }^{2}$ and Timothy Booth ${ }^{2}$ \\ ${ }^{1}$ Institute for Biodiagnostics, National Research Council Canada, 435 Ellice Ave., Winnipeg, Canada, R3B 1Y6 \\ and ${ }^{2}$ National Microbiology Laboratory, Canadian Science Center for Human and Animal Health, 1015 \\ Arlington Street, Winnipeg, Manitoba,Canada R3E 3M4
}

Given that the infrared spectrum of most materials is one of the most characteristic properties of that material, it seems reasonable to assume that infrared spectra of human tissues will be equally characteristic. This has in fact been shown to be the case, with many reports appearing in the literature over the last 10 years that describe the spectral characteristics of both normal and abnormal tissues.

A consensus is now appearing that IR spectroscopic analysis of tissues is most reliably performed using an infrared microscope. This consensus is based upon the fact that IR microscopy allows tissues to be probed no n-destructively with relatively high spatial resolution and direct correlation may be made between spectra and sample histology. However, there is by no means consensus with regards to sample preparation and data acquisition methods.

A number of important questions remain to be addressed before such a consensus can be reached. For example, is it realistic to analyse tissues that have been stained? What is the most appropriate instrumental approach (standard mapping microscopy, synchrotron based microscopy, microscopic imaging with a focal plane array system)? These questions will be addressed. Results from studies on a variety of tissues will be presented that demonstrate that significant spectral changes may be observed in tissues that have undergone the various manipulations involved in staining. For example the first stage in staining, exposure to alcohol/water, results in a decreased lipid content and removal of glycogen from tissues sections (Figure 1). These changes may be directly attributed to simpl e phenomena such as dissolution of materials in the staining media.

The potential advantages and disadvantages of infrared microscopy performed using three distinct approaches will be discussed. Data acquired by conventional infrared microscopy, synchrotro n-based microscopy and microscopic imaging with a focal plane array detection system will be presented. Conventional microscopy has the advantage of simplicity, but is limited to a spatial resolution of around $25 \mu \mathrm{m}$. The use of a synchrotron source can significantly improve the achievable spatial resolution $(5 \mu \mathrm{m}$ or better, see Figure 2) but access may be problematic. Microscopic imaging using focal plane array detection holds the promise of improved spatial resolution and decreased measurement times but signal to noise may be compromised.

Choosing the appropriate measurement technique clearly involves compromise and requires an understanding of the strengths and weaknesses of each technique. The three techniques will be compared and contrasted using data obtained from skin tissue. Issues such as signal to noise, spatial resolution, speed and volume of data will be addressed. 


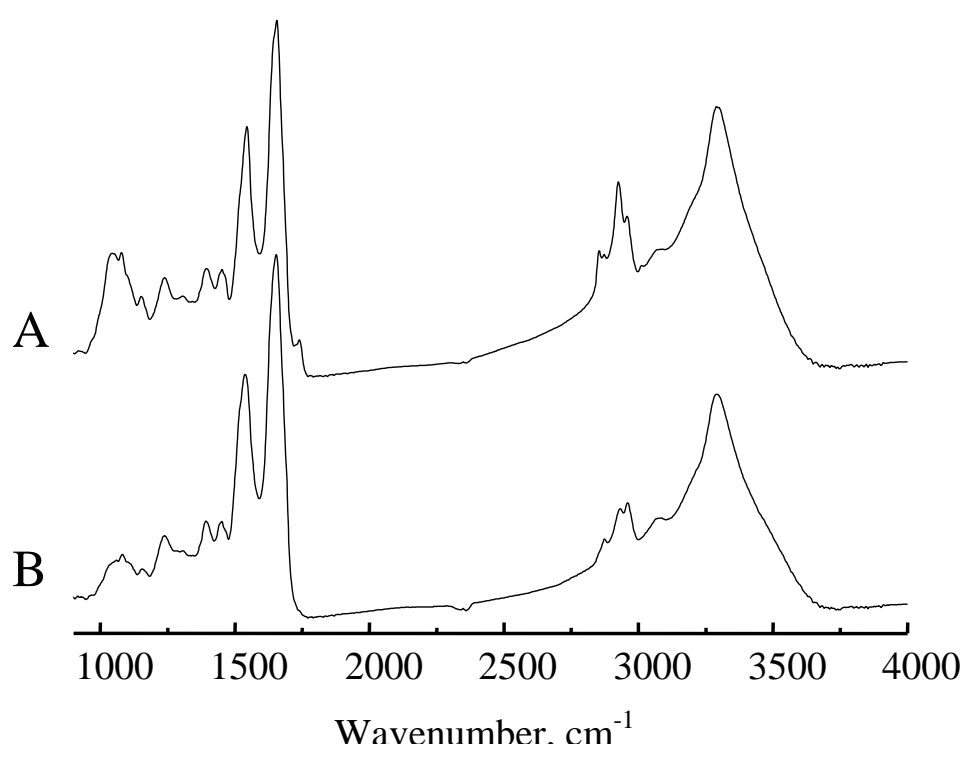

Figure 1. Infrared spectra of mouse liver before (A) and after (B) the first step in H\& E staining (immersion in $100 \%$ alcohol followed by $95 \%$ alcohol followed by water).

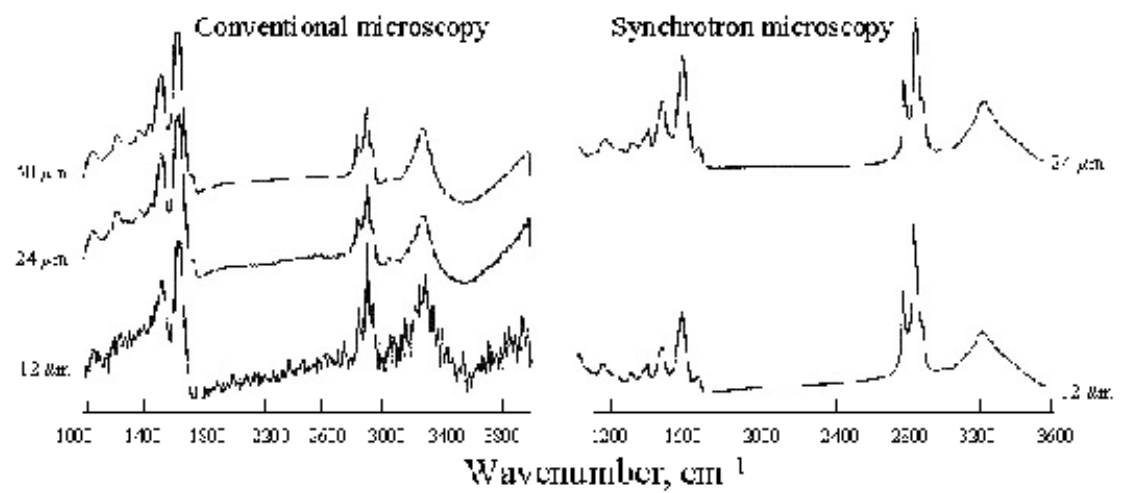

Figure 2. A comparison of spectra of human CNS tissue obtained at various aperture sizes using conventional and synchrotron based infrared microscopy.

\section{References:}

"Vibrational Spectroscopy and Pathology" M. Jackson and H. H. Manstch in Handbook of Vibrational Spectroscopy, in press.

"Medical Science Applications of Infrared Spectroscopy" Jackson, M. and Mantsch, H.H. in Encyclopedia of Spectroscopy and Spectrometry, Vol. 2, 1271-1281, 2000.

"Analysis and interpretation of infrared microscopic maps: visualisation and classification of skin components by digital staining and multivariate analysis" Mcintosh, L M., Mansfield, J.R., Crowson, A. N,. Mantsch, H.H. and Jackson, M. Biospectroscopy, 5, 265-275, 1999

"A LDA-guided search engine for the non-subjective analysis of infrared microscopic maps" Mansfield, J.R., McIntosh, L M., Crowson, A. N., Mantsch, H.H. and Jackson, M. Appl. Spectrosc. 53, 1323-1330, 1999

"In-Situ characterization of $\beta$-amyloid in Alzheimer's diseased tissue by synchrotron FTIR microspectroscopy" Choo, L.-P., Wetzel, D.L., Halliday, W.C., Jackson, M., LeVine, S.M. and Mantsch, H.H. Biophys J. 71, 1672-1679, 1996. 